\title{
The effect-enhancing and toxicity-reducing activity of Hypericum japonicum Thunb. extract in murine liver cancer chemotherapy
}

\author{
HONG-BO ZHANG ${ }^{1,3}$, PING LU $^{2}$, WEN-BO CAO ${ }^{3}$, ZHEN-HUA ZHANG $^{1}$ and XIANG-LEI MENG ${ }^{3}$ \\ ${ }^{1}$ Department of Anatomy and Cell Biology, School of Medicine, Zhengzhou University, Zhengzhou, Henan 450001; \\ ${ }^{2}$ School of Pharmacy, Henan University of Traditional Chinese Medicine, Zhengzhou, Henan 450008; \\ ${ }^{3}$ Department of Cancer Biology, School of Medicine, Zhengzhou University, Zhengzhou, Henan 450001, P.R. China
}

Received September 18, 2012; Accepted December 11, 2012

DOI: $10.3892 / \operatorname{mco} .2012 .52$

\begin{abstract}
Chinese herbs are potential sources of antitumor drugs with immunoregulatory activity and few adverse effects. In the present study, we investigated whether the Hypericum japonicum Thunb. (HJT) extract enhanced the efficacy of 5-fluorouracil (5-FU) treatment in murine liver tumor xenografts and reduced toxicity of chemotherapy in hepatoma H22-bearing mice. Tumor weight and inhibition rate, thymus and spleen indices, as well as white blood cell (WBC) count were calculated. The phagocytic function of macrophages was assessed by observing peritoneal macrophages phagocytized chicken red blood cells (RBC). Body weight and toxic reactions of the chemotherapeutic and life prolongation rate were investigated in the mice. Results demonstrated that the HJT extract significantly enhanced the tumor inhibition rate of 5-FU, improved the immune function, reduced the toxic effects and prolonged the survival time in the tumor-bearing mice. Taken together, these results indicated that the HJT extract has a synergistic tumor-inhibiting effect with 5-FU, is able to reduce the toxic side effects and is likely to be safe and efficacious for use in antitumor therapy.
\end{abstract}

\section{Introdution}

Liver cancer is a malignant disease with a high incidence and mortality, and is often diagnosed at an advanced stage (1). Common therapies, such as surgical resection, percutaneous or transarterial interventions are of limited efficacy (2), as chemotherapy, one of the main measures for a comprehensive treatment, may induce adverse effects such as bone marrow

Correspondence to: Dr Hong-Bo Zhang, Department of Anatomy and Cell Biology, School of Medicine, Zhengzhou University, 100 Kexue Road, Zhengzhou, Henan 450001, P.R. China

E-mail: zhanghongbo1975@gmail.com

Key words: antitumor, Hypericum japonicum Thunb., hepatoma, chemotherapy, mice suppression, gastrointestinal reactions and lowered immunological function.

Several Chinese herbs have been identified as potential sources of antitumor drugs (3-5). The effect-enhancing and toxicity-reducing activity of Chinese herbs in radiotherapy and chemotherapy of tumors was previously confirmed $(6,7)$. However, no studies focusing on the antitumor and toxicity-reducing effects of Hypericum japonicum Thunb. (HJT) have been reported thus far. Therefore, the present investigation was conducted to ascertain whether the HJT extract enhanced the efficacy of 5-fluorouracil (5-FU) treatment in murine liver tumor xenografts, and reduced toxicity of chemotherapy in tumor-bearing mice.

\section{Materials and methods}

Reagents. RPMI-1640 medium, fetal bovine serum (FBS), penicillin $\mathrm{G}$ and streptomycin were obtained from Invitrogen Corp. (Carlsbad, CA, USA). 5-FU was obtained from the Shanghai Xudong Haipu Pharmaceutical Co., Ltd. (Shanghai, China). Murine H22 hepatoma cells were purchased from the Shanghai Institute of Cell Biology at the Chinese Academy of Sciences (Shanghai, China).

Animals. Institute of Cancer Research (ICR) mice (male/female, 50-50\%; weight, 20 $\pm 2 \mathrm{~g}$ ) were purchased from the Experimental Animal Center, Zhengzhou University (Zhengzhou, China). The animals were kept in a standard laboratory, under a 12-h light/dark cycle, at room temperature $\left(20 \pm 2^{\circ} \mathrm{C}\right)$, in a humid $(75 \pm 15 \%)$ and filtered laminar air flow-controlled room in the animal facility of the Experimental Animal Center of the Zhengzhou University. Mice were raised and cared for, given autoclaved water and fed ad libitum with laboratory pellet chow. Experiments were performed according to the regulations and guidelines for animal experiments in China. This study was approved by the Ethics Committee of Zhenghou University, Zhenghou, China.

Cell culture. H22 cells were cultured in RPMI-1640 medium supplemented with $10 \% \mathrm{FBS}, 1 \times 10^{5} \mathrm{U} / 1$ penicillin and $100 \mathrm{mg} / 1$ streptomycin in a humidified atmosphere with 
Table I. Effect of the HJT extract on body and tumor weight of the H22-bearing mice [mean \pm standard deviation (SD), n=10].

\begin{tabular}{|c|c|c|c|c|c|c|}
\hline \multirow[b]{2}{*}{ Group } & \multirow{2}{*}{$\begin{array}{c}\text { Dose } \\
\text { (g/kg/day) }\end{array}$} & \multicolumn{2}{|c|}{ Body weight (g) } & \multirow{2}{*}{$\begin{array}{c}\text { Variation of body } \\
\text { weight }(\mathrm{g})\end{array}$} & \multirow{2}{*}{$\begin{array}{c}\text { Tumor weight } \\
(\mathrm{g})\end{array}$} & \multirow{2}{*}{$\begin{array}{c}\text { Tumor inhibition } \\
\text { rate }(\%)\end{array}$} \\
\hline & & Pre-treatment & Post-treatment & & & \\
\hline Normal control & - & $20.05 \pm 2.07$ & $24.35 \pm 3.24$ & $4.30 \pm 3.47^{\mathrm{a}, \mathrm{b}}$ & - & - \\
\hline Tumor control & - & $20.11 \pm 1.94$ & $21.07 \pm 2.02$ & $0.96 \pm 2.13$ & $2.70 \pm 1.13$ & - \\
\hline \multirow[t]{2}{*}{$5-\mathrm{FU}$} & 0.02 & $19.98 \pm 2.03$ & $20.55 \pm 1.86$ & $0.57 \pm 1.89^{\mathrm{a}}$ & $1.59 \pm 0.59^{\mathrm{a}}$ & 41.11 \\
\hline & $0.02+3.00$ & $20.01 \pm 2.25$ & $21.49 \pm 1.94$ & $1.48 \pm 1.65^{\mathrm{a}, \mathrm{b}}$ & $1.23 \pm 0.54^{\mathrm{a}, \mathrm{b}}$ & 54.44 \\
\hline \multirow[t]{2}{*}{$5-\mathrm{FU}+\mathrm{HJT}$} & $0.02+6.00$ & $20.07 \pm 2.06$ & $22.15 \pm 1.75$ & $2.08 \pm 2.03^{\mathrm{a}, \mathrm{b}}$ & $1.13 \pm 0.42^{\mathrm{a}, \mathrm{b}}$ & 58.15 \\
\hline & $0.02+12.00$ & $19.96 \pm 1.95$ & $23.24 \pm 2.05$ & $3.28 \pm 2.19^{\mathrm{a}, \mathrm{b}}$ & $0.91 \pm 0.63^{\mathrm{a}, \mathrm{b}}$ & 66.29 \\
\hline
\end{tabular}

${ }^{a} \mathrm{P}<0.05$ vs. tumor control group; ${ }^{\mathrm{b}} \mathrm{P}<0.05$ vs. the 5-FU group. HJT, Hypericum japonicum Thunb.

$5 \% \mathrm{CO}_{2}$ incubator at $37^{\circ} \mathrm{C}$. The cells were subcultured until logarithmic growth phase was reached.

Preparation of the HJT extract. HJT extract was obtained from Xi'an Kanger America Biotechnology Co., Ltd. (Xi'an, China). Air-dried HJT samples were ground and mixed with double-distilled water at $100^{\circ} \mathrm{C}$ for $10 \mathrm{~min}$, then cooled to room temperature. HJT was then stored at $4^{\circ} \mathrm{C}$ in dark bottles and kept sterile until instilled intragastrically in the mice.

Experimental design. ICR mice were randomized into A-F groups $(n=10)$. The animals were subcutaneously injected with $\mathrm{H} 22$ cells ( $1 \times 10^{7}$ cells/mouse) into the armpit of the right hind limb, with the exception of group A (normal group), which received sodium suspension (0.9\%). Group B served as the tumor control. In groups C, D, E and F, 5-FU (20 mg/kg) was injected into the abdominal cavity, once daily. Groups D (low-dose HJT group), E (medium-dose HJT group) and F (high-dose HJT group) received perfusion of HJT extract intragastrically at 3, 6 and $12 \mathrm{~g} / \mathrm{d}$ per $\mathrm{kg}$, administered $24 \mathrm{~h}$ after the tumor inoculation, once daily for 10 days.

Determination of antitumor activity in vivo. The general condition of mice such as movement, fur, eating, drinking, body weight increase and toxic responses was examined. Twenty four hours after the last dose, blood was collected from the animals for the white blood cell (WBC) count by retro-orbital puncture under slight anesthesia (diethyl ether). Mice from each group were sacrificed under anesthesia using $\mathrm{CO}_{2}$. The mice and the separated tumor were weighed. Antitumor activity was evaluated by tumor weighing. The tumor inhibition rate was calculated as: Tumor inhibitory rate $(\%)=(1$-average tumor weighing of administration group/average tumor weighing of the tumor control group) x $100 \%$ (9). Simultaneously, the thymus and spleen were excised and weighed. Indices were calculated as: Thymus index $=$ the thymus weight $(\mathrm{mg}) /$ body weight $(\mathrm{g})$ and spleen index $=$ the spleen weight $(\mathrm{mg}) /$ body weight (g) (10).

Determination of the phagocytic function of macrophage. After the last dose and 12-h fasting, chicken-red cells $(1 \mathrm{ml}$, $5 \%$ ) were injected intraperitoneally into each group. After $12 \mathrm{~h}$, the mice were sacrificed and normal saline $(2 \mathrm{ml})$ was
A

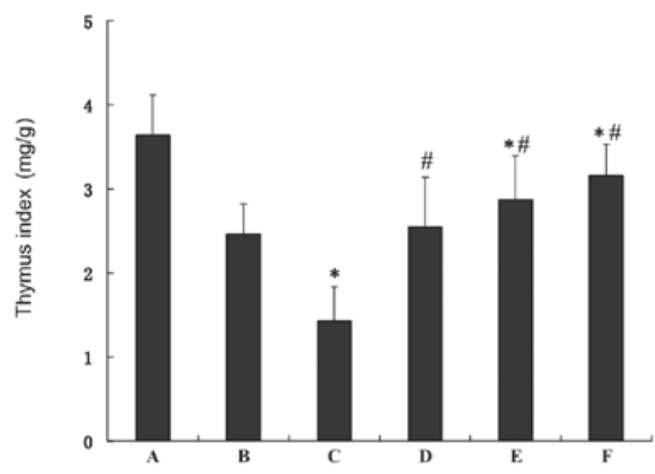

B

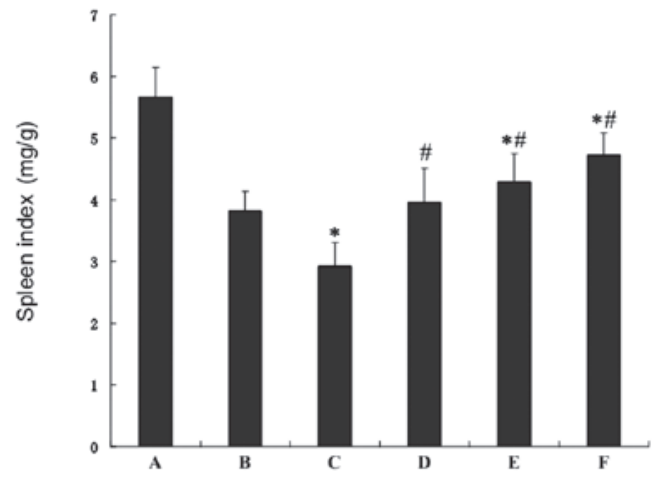

C

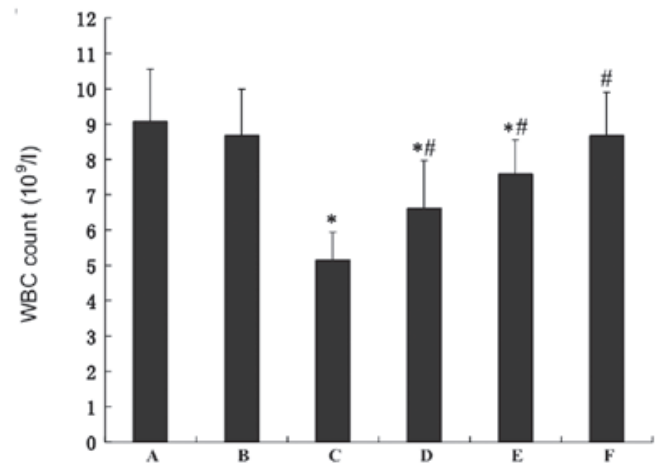

Figure 1. Effect of Hypericum japonicum Thunb. (HJT) extract on immune organs and white blood cell (WBC) count of H22-bearing mice. Group A, normal control; Group B, tumor control; Group C, 5-FU; Group D, 5-FU + low-dose HJT; Group E, 5-FU + medium-dose HJT; Group F, 5 -FU + high-dose HJT. Results are presented as the mean \pm standard deviation for the animals in each group. ${ }^{*} \mathrm{P}<0.05$ vs. tumor control group; ${ }^{*} \mathrm{P}<0.05$ vs. 5-FU group. 
Table II. Effects of the HJT extract on the life prolongation rate in the H22-bearing mice [mean \pm standard deviation (SD), $\mathrm{n}=10$ ].

Life prolongation rate $(\%)$

\begin{tabular}{lcccc}
\cline { 3 - 4 } Group & Dose $(\mathrm{g} / \mathrm{kg} /$ day $)$ & Survival time $($ day $)$ & vs. tumor control group & vs. 5-FU group \\
\hline Tumor control & - & $15.98 \pm 4.85$ & - & - \\
$5-F U$ & 0.02 & $16.23 \pm 4.03$ & 1.56 & 24.03 \\
& $0.02+3.00$ & $20.13 \pm 3.89^{\mathrm{a}, \mathrm{b}}$ & 25.97 & 41.53 \\
$5-\mathrm{FU}+$ HJT & $0.02+6.00$ & $22.97 \pm 4.31^{\mathrm{a}, \mathrm{b}}$ & 43.74 & 59.77 \\
& $0.02+12.00$ & $25.93+5.07^{\mathrm{a}, \mathrm{b}}$ & 62.27 & 59 \\
\hline
\end{tabular}

${ }^{\mathrm{a}} \mathrm{P}<0.05$ vs. tumor control group; ${ }^{\mathrm{b}} \mathrm{P}<0.05$ vs. the 5-FU group. HJT, Hypericum japonicum Thunb.

injected into the abdominal cavity. Peritoneal fluid $(1 \mathrm{ml})$ was then drawn for glass slides. Following incubation for $30 \mathrm{~min}$, peritoneal fluid was fixed with a mixture of acetone/methanol $(1: 1, v / v)$ and stained with $4 \%$ Giemsa. After drying, peritoneal macrophages were counted by microscopy. The effect of the HJT extract on phagocytosis of enterocoelia macrophage was evaluated by the chicken-red cell phagocytic rate and index, calculated using the formulas: phagocytic index = chicken-red cells phagocyted/total macrophages and phagocytic rate = (macrophages that phagocytized red blood cells (RBC)/total macrophages) $\times 100 \%$ (11).

Determination of life prolongation rate in the H22-bearing mice. The grouping and drug administration for this experiment were the same as described above $(n=10)$. H22 cells $\left(1 \times 10^{7}\right.$ cells/mouse) were injected into the abdominal cavity of each mouse. The survival time of the mice was recorded for 35 days after terminating drug administration. The experiment was considered invalid if the mortality rate in the control group during the experiment was $>20 \%$, or the survival time of $20 \%$ mice was $>5$ weeks. The life prolongation rate was determined as (the mean living days of the mice in the treatment group/the mean living days in the tumor control group-1) x100\%.

Statistical analysis. Values were expressed as the mean \pm standard deviation (SD). Statistical analysis was performed with one-way analysis of variance (ANOVA) using the statistical software SPSS 17.0. $\mathrm{P}<0.05$ was considered to indicate a statistically significant difference.

\section{Results}

Antitumor and toxicity-reducing activity of the HJT extract on hepatoma. H22-bearing mice in the tumor control group were not lively, with dark fur, and exhibited loss of appetite as well as slow weight gain. Additionally, mice in the 5-FU group died prior to the last administration. By contrast, mice in the HJT extract groups were vigorous, with shiny fur and a rapid body weight increase, showing a good condition. The toxic effects in the 5-FU group were severe, including anorexia, abdominal distention and emaciation, while the mice in the HJT extract groups showed no or little toxicity.
No statistically significant difference was detected in the body weight between each group prior to treatment $(\mathrm{P}>0.05)$ (Table I). Following the experiment, the body weight of the tumor-bearing mice in the groups increased to varying degrees. The body weight increase was significantly higher in each HJT group compared with the tumor control group $(\mathrm{P}<0.05)$, whereas the increase in the 5-FU group was significantly lower compared with the other groups $(\mathrm{P}<0.05)$. The tumor weight of three HJT groups and the 5-FU group was significantly lower compared with the tumor control group $(\mathrm{P}<0.05)$. Compared with the 5-FU group, the tumors of three HJT groups showed a significant decrease in size, with the HJT dose group increasing $(\mathrm{P}<0.05)$. The tumor inhibitory rates of the 5-FU group and HJT groups were 41.11, 54.44, 58.15 and $66.29 \%$, respectively.

Effect of the HJT extract on immune organs and WBC count of H22-bearing mice. As shown in Fig. 1, compared with the normal control group, the thymus and spleen indices were significantly lower in the tumor control group and further decreased in the 5-FU group $(\mathrm{P}<0.05)$. In the HJT extract groups, atrophy of the immune organs induced by 5-FU was improved $(\mathrm{P}<0.05)$. Compared with the tumor control group, the WBC count showed a significant decrease in the 5-FU group $(\mathrm{P}<0.05)$, and showed an increasing tendency in the HJT extract groups.

Effect of the HJT extract on the phagocytic function of macrophages in H22-bearing mice. Compared with the tumor control group, 5-FU decreased the phagocytic index and rate significantly $(\mathrm{P}<0.05)$ (Fig. 2). However, the phagocytic function of the macrophages of mice was increased in the HJT groups. The phagocytic indices of the low-, medium- and highdose HJT groups were increased 16.32, 53.31 and $81.63 \%$, respectively, compared with the 5-FU group. The phagocytic rates of the low-, medium- and high-dose HJT groups were increased by $35.07,54.47$ and $83.31 \%$, respectively, compared with the 5-FU group.

Effects of the HJT extract on the life prolongation rate in H22-bearing mice. As shown in Table II, the survival time of the ascites tumor mice in the HJT groups was significantly higher compared with those of the tumor control $(\mathrm{P}<0.05)$ and 
A

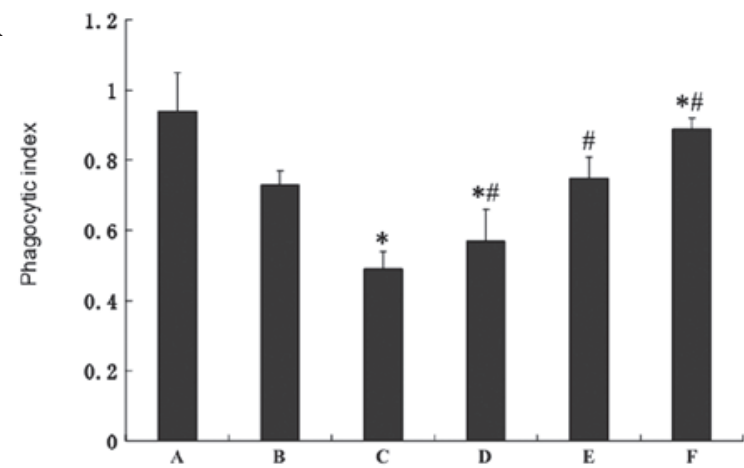

B

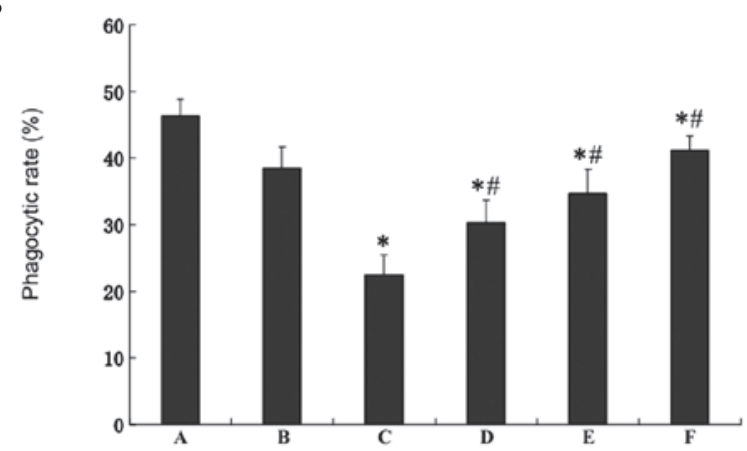

Figure 2. Effect of Hypericum japonicum Thunb. (HJT) extract on the phagocytic function of macrophages in H22-bearing mice. Group A, normal control; Group B, tumor control; Group C, 5-FU; Group D, 5-FU + low-dose HJT; Group E, 5-FU + medium-dose HJT; Group F, 5-FU + high-dose HJT. Results are presented as the mean \pm standard deviation for the animals in each group. ${ }^{\prime} \mathrm{P}<0.05$ vs. tumor control group; ${ }^{~} \mathrm{P}<0.05$ vs. 5 -FU group.

5-FU groups $(\mathrm{P}<0.05)$. Survival time of the 5-FU group was slightly higher compared with that of the tumor control group, with no significant difference being detected between the two groups. The life prolongation rates in the low-, medium- and high-dose HJT groups were 25.97, 43.74 and $62.27 \%$, respectively, which were significantly higher compared with those of the 5-FU group.

\section{Discussion}

Previous studies have noted the bioactivies of the Hypericum genus including the antitumor, antioxidant, antimicrobial and natural killer (NK) cell-activating activity (8-12). HJT, an annual herb from the genus Hypericum L., known in traditional Chinese medicine as Tian-ji-huang, grows mainly throughout southern China. HJT has been used in a Chinese herbal compound formula for many years for the treatment of bacterial diseases; infectious, acute and chronic hepatitis; gastrointestinal disorder; internal hemorrhage and tumors $(13,14)$. Data in the present study demonstrated that the HJT extract significantly inhibited the growth of $\mathrm{H} 22$-transplanted tumors and had a synergistic tumor-inhibiting effect with the 5-FU group.

Reduction of the chemotherapy-induced side effects in cancer treatment has gained increasing attention. The results of this study demonstrated that the HJT extract exhibited an improved efficacy of supporting treatment in chemotherapy. The increase in body weight markedly reduced toxic reactions and the higher life prolongation rate demonstrated that the HJT extract had toxicity-reducing activity.

The immune function is important in the course of radiotherapy and chemotherapy for patients with malignant tumors. Findings of the present study have shown that the thymus and spleen indices, WBC count and the phagocytic function were elevated in the HJT groups, indicating that the HJT extract efficaciously antagonized the immunosuppression caused by 5 -FU and may be used as an immune response modifier in chemotherapy. It is possible to estimate that HJT likely improves the immune function of patients suffering from a malignant disease.

The antitumor and toxicity-reducing effects of HJT extract are potentially associated with components such as flavonoids, xanthones, glucoside and embelin in HJT, which also contribute to the immune activity on the tumor cells (15-18). However, in the present study, the whole herb extract was used, while the concrete mechanism of action remains to be elucidated.

\section{Acknowledgements}

This study was supported by the Talent Recruitment Research Grant of the Zhengzhou University, Zhengzhou, China.

\section{References}

1. Jemal A, Bray F, Center MM, Ferlay J, Ward E and Forman D: Global cancer statistics. CA Cancer J Clin 61: 69-90, 2011.

2. Dai LC, Wang X, Yao X, Lu YL, Ping JL and He JF: Enhanced therapeutic effects of combined chemotherapeutic drugs and midkine antisense oligonucleotides for hepatocellular carcinoma. World J Gastroenterol 13: 1989-1994, 2007.

3. Yu J, Liu H, Lei J, Tan W, Hu X and Zou G: Antitumor activity of chloroform fraction of Scutellaria barbata and its active constituents. Phytother Res 21: 817-822, 2007.

4. Vickers A: Botanical medicines for the treatment of cancer: rationale, overview of current data, and methodological considerations for phase I and II trials. Cancer Invest 20: 1069-1079, 2002.

5. Liu F, Wang JG, Wang SY, Li Y, Wu YP and Xi SM: Antitumor effect and mechanism of Gecko on human esophageal carcinoma cell lines in vitro and xenografted sarcoma 180 in Kunming mice. World J Gastroenterol 14: 3990-3996, 2008.

6. Wu SL, Sun ZJ, Yu L, Meng KW, Qin XL and Pan CE: Effect of resveratrol and in combination with 5-FU on murine liver cancer. World J Gastroenterol 10: 3048-3052, 2004.

7. Cao Y, Xia QH, Meng H and Zhong AP: Antitumor and synergistic effect of Chinese medicine 'bushen huayu jiedu recipe' and chemotherapy on transplanted animal hepatocarcinoma. World J Gastroenterol 11: 5218-5220, 2005.

8. Dongre SH, Badami S, Natesan S and H RC: Antitumor activity of the methanol extract of Hypericum hookerianum stem against Ehrlich ascites carcinoma in Swiss albino mice. J Pharmacol Sci 103: 354-359, 2007.

9. Radulović N, Stankov-Jovanović V, Stojanović G, Šmelcerović A, Spiteller M and Asakawa Y: Screening of in vitro antimicrobial and antioxidant activity of nine Hypericum species from the Balkans. Food Chem 103: 15-21, 2007.

10. Jayasuriya H, Clark AM and McChesney JD: New antimicrobial filicinic acid derivatives from Hypericum drummondii. J Nat Prod 54: 1314-1320, 1991.

11. Jayasuriya H, McChesney JD, Swanson SM and Pezzuto JM: Antimicrobial and cytotoxic activity of rottlerin-type compounds from Hypericum drummondii. J Nat Prod 52: 325-331, 1989.

12. Helgason CM, Frank JL, Johnson DR, Frank MG and Hendricks SE: The effects of St. John's Wort (Hypericum perforatum) on NK cell activity in vitro. Immunopharmacology 46: 247-251, 2000.

13. Ishiguro K, Yamaki M, Kashihara M and Takagi S: Sarothralen A and $\mathrm{B}$, new antibiotic compounds from Hypericum japonicum. Planta Med 52: 288-290, 1986. 
14. Wu QL, Wang SP, Du LJ, Yang JS and Xiao PG: Xanthones from Hypericum japonicum and $H$. henryi. Phytochemistry 49: $1395-1402,1998$

15. Wang XW, Mao Y, Wang NL and Yao XS: A new phloroglucinol diglycoside derivative from Hypericum japonicum Thunb. Molecules 13: 2796-2803, 2008.

16. Dai Y, Desano J, Qu Y, Tang W, Meng Y, Lawrence TS and Xu L: Natural IAP inhibitor Embelin enhances therapeutic efficacy of ionizing radiation in prostate cancer. Am J Cancer Res 1: $128-143,2011$
17. Ding M, Zhao J, Bowman L, Lu Y and Shi X: Inhibition of AP-1 and MAPK signaling and activation of Nrf2/ARE pathway by quercitrin. Int J Oncol 36: 59-67, 2010.

18. Lin JP, Yang JS, Lu CC, Chiang JH, Wu CL, Lin JJ, Lin HL, Yang MD, Liu KC, Chiu TH and Chung JG: Rutin inhibits the proliferation of murine leukemia WEHI-3 cells in vivo and promotes immune response in vivo. Leuk Res 33: 823-828, 2009. 